\title{
A Model Driven Framework for Geographic Knowledge Discovery
}

\author{
Octavio Glorio \\ Department of Software \\ and Computing Systems \\ University of Alicante \\ Alicante, PO BOX 99 E-03080 \\ Email: oglorio@dlsi.ua.es
}

\author{
Jose Zubcoff \\ Department of Sea Sciences \\ and Applied Biology \\ University of Alicante \\ Alicante, PO BOX 99 E-03080 \\ Email: jose.zubcoff@ua.es
}

\author{
Juan Trujillo \\ Department of Software \\ and Computing Systems \\ University of Alicante \\ Alicante, PO BOX 99 E-03080 \\ Email: jtrujillo@dlsi.ua.es
}

\begin{abstract}
Geographic knowledge discovery (GKD) is the process of extracting information and knowledge from massive georeferenced databases. Usually the process is accomplished by two different systems, the Geographic Information Systems (GIS) and the data mining engines. However, the development of those systems is a complex task due to it does not follow a systematic, integrated and standard methodology. To overcome these pitfalls, in this paper, we propose a modeling framework that addresses the development of the different parts of a multilayer GKD process. The main advantages of our framework are that: (i) it reduces the design effort, (ii) it improves quality systems obtained, (iii) it is independent of platforms, (iv) it facilitates the use of data mining techniques on geo-referenced data, and finally, (v) it ameliorates the communication between different users.
\end{abstract}

\section{INTRODUCTION}

Geographic knowledge discovery (GKD) is the process of extracting information and knowledge from massive georeferenced databases. Usually, the process is taken by two isolated systems: the Geographic Information Systems (GIS) and the data mining engines. There are also some ad hoc processes that are taken by integrated systems that merge these two technologies. However, the development on such systems is a complex task mainly because it does not follow a systematic, integrated and standard methodology.

Data mining needs the geographic data available and, furthermore, prepared to be analyzed. Moreover, the repository must contain the right data (geographic and not geographic) in order to have a successful GKD. In order to improve the data quality the geographic data must be available in the repository and this can be done at early stages of the Data Warehouse (DW) development: from the conceptual modeling of DW.

In this paper, we propose a modeling framework that addresses the development of the different parts of a multilayer GKD process. To accomplish this task, we have defined the spatial repository layer, the geographic customization layer and the application layer represented by different data mining techniques. Therefore, we have described how to build the different conceptual models by using the profile extension of the Unified Modeling Language (UML). In order to show the suitability of our propose, we have modeled an actual case of study that handles a very large database that contains geo-referenced data about the abundance of commercial fish species around marine protected areas. For this purpose, we have also developed an Eclipse based prototype that implements our developing methodology and delivers platform specific code.

The main advantages of our framework are (i) the dramatic reduction of the design effort due to automatics process are able to deliver specific platform code for the different process layers, (ii) the high quality systems obtained while good practices are reused in the different modeling tasks, (iii) the ability for adapting to new platform technologies while these tasks are entrusted to the model-to-model transformation scaffolding, and (iv) the suitability for the use of data mining techniques on geo-referenced data, avoiding isolated GIS and data mining session and supporting the documentation using intuitive visual models. Furthermore, the communication between the different users of the process is facilitated while using a formal and standard language to describe the different models.

\section{A. Running Example}

Throughout the paper we will be focus on real case of study. The main objective is the coastal fisheries management and the use of Marine Protected Areas (MPA). These locations were impacts are been managed, have important ecological and socio-economical benefits, being used to protect habitats, biological richness, restore fishing stocks, and conserve fish populations and despoiled areas [1]. In order to obtain knowledge about the impact of the MPA it is needed some geographical data prepared in the repository. (i) The distance to the next MPA is an important measure for the use of MPA networks. (ii) The distances to the main city, or to a main airport are other measures that can be used in the analysis. (iii) The size of the protected area (perimeter) can be used to obtain important information about the effect of the sizes of the different MPAs.

\section{RELATED WORK}

There are many works related to conceptual modeling for knowledge discovery systems. Some of the most well-known approaches are following cited. In [1], Bimonte et al. propose a multidimensional model (GeoCube) which integrates 
geographic information and ensure correct aggregation over this kind of measures. Then, they present GeWOlap [2], an extended model that support a web implementation of SOLAP tools. Finally in [3], they propose the extension of the traditional spatial dimensions to support complex map navigation. Gomez et al. [4] also define a formal model for representing spatial data and a GIS-OLAP tool named Piet [5]. This model identify three parts in spatial dimensions, the OLAP part, the geometric part and the algebraic part. It also supports complex algorithms for improve query performance.

Fidalgo et al. [6] propose a formal model named GeoDWFrame for guiding the designing of GDWs. Then, some extensions were made to this framework by the authors where a formal metamodel for GDW is defined [7] and a set of aggregation functions for spatial measures where developed [8]. Finally, they have presented a geographic and multidimensional data cube metamodel and a query language named GeoMDQL [9], which allows simultaneous usage of both multidimensional and spatial operators.

On the other hand, present work is based on our previous framework defined in [10]. This work align the design of every layer of a spatial data warehouse, using the three MDA viewpoints. Unfortunately, geographic information do not full fit on available spatial multidimensional elements (spatial levels and measures) and geographic capabilities over data cubes were not considered.

\section{MDA FOUNDATIONS}

The architecture of data warehouses is usually depicted as a set of components arranged in five layers (see Fig. 1). The design of every layer has its own characteristics and pitfalls. On the other hand, MDA encourages the system development by specifying a Platform Independent Model (PIM) which contains no specific information of the platform or the technology that is used to realize it. Then, this PIM can be transformed into a Platform Specific Model (PSM) in order to include information about the specific technology that is used in the implementation on a specific platform.

Following these considerations, in present work, we have defined a model driven approach to accomplish geo-referenced repositories generation, geographic customized data cubes and data mining classification sessions. In Fig. 1, we describe schematically our framework. The development process start with the definition of a multidimensional model able to represent correctly geo-referenced data (GeoMD PIM). Then, a model which it is able to represent geographic operations over data cubes (GeoCustom PIM) is generated by using a model to model transformation set (T3). The obtained model is manipulated by analyst in order to define complex operations over data cubes using the avaiable geo-referenced data. Finally, a data mining classification sesion over the resultant data cube is designed using a specific data mining conceptual model (Classification PIM).

PIMs and PSMs can be specified by using any modeling language, but typically MOF-compliant languages. In our approach, we use UML ${ }^{1}$ for PIMs definition since it is a standard modeling language for general purpose and it can be extended to define specialized languages for certain domains (i.e. metamodel extensibility or profiles). In order to define PSMs, we use CWM ${ }^{2}$ as modeling language because its Resource metamodel package is a standard to represent the structure of data according to several different database technologies, such as relational or multidimensional. In order to delivered platform specific code, transformations between models ( $\left.\mathrm{T}_{1}, \mathrm{~T} 3, \mathrm{~T} 4\right)$ and final code mappings ( $\left.\mathrm{T} 2, \mathrm{~T} 5, \mathrm{~T} 6\right)$ had been developed. Required transformation sets are formally established by using QVT (Query/Views/Transformations) and MOFScript in order to define readable, understandable, adaptable, maintainable, and automatic transformations between MOF-compliant models. Due to space constraints, in this paper we focus on the modeling tasks either on transformation sets because their importance and relevance in the proposed framework.

\section{Geographic Multidimensional Model}

In Fig. 1 it is shown the model defined in this section (GeoMD PIM), it basically encourage the representation of geo-referenced data on multidimensional structures. This model is based on a multidimensional model [11] and an spatial extension [10] presented for us. This multidimensional model organize elegantly non spatial information into facts and dimensions. These facts and dimensions are modeled by Fact (W) and Dimension ( of measures or attributes corresponding to FactAttribute (FA) stereotype. With respect to dimensions, each aggregation level of a hierarchy is specified by classes stereotyped as Base $([\boldsymbol{B})$. Then, in [10], we introduced a new element as a hierarchy level with a geometry associated (spatial level). This element is used for correct aggregation over partially contained hierarchy levels. We have also introduced a spatial measures element for support multidimensional analysis over geometric objects. These spatial level and spatial measure are modeled by SpatialLevel ( $\left(^{*}\right)$ and SpatialMeasure (*) stereotypes.

In this section, we propose to introduce the geographic information in the previous described spatial multidimensional model. Geographic information is spatial data referenced to the Earth. These complex data have been represented, in [10] using spatial levels. Therefore, spatial levels are possible suitable elements for representing geographic information, they are able to represent thematic attributes and a geometric description. However, levels (spatial or not) are related to the multidimensional model by semantics constraints codified in Object Constraint Language (OCL). For example, levels can only be associated with dimensions or levels. On the other side, geographic information is not associated with the multidimensional elements by these semantics, these data are just related with all other data that coexist in the same reference system. This is the main reason to create another

\footnotetext{
${ }^{1}$ Object Management Group, Unified Modeling Language (UML) 2.1.1

${ }^{2}$ Object Management Group, Common Warehouse Metamodel (CWM) 1.0 .1
} 


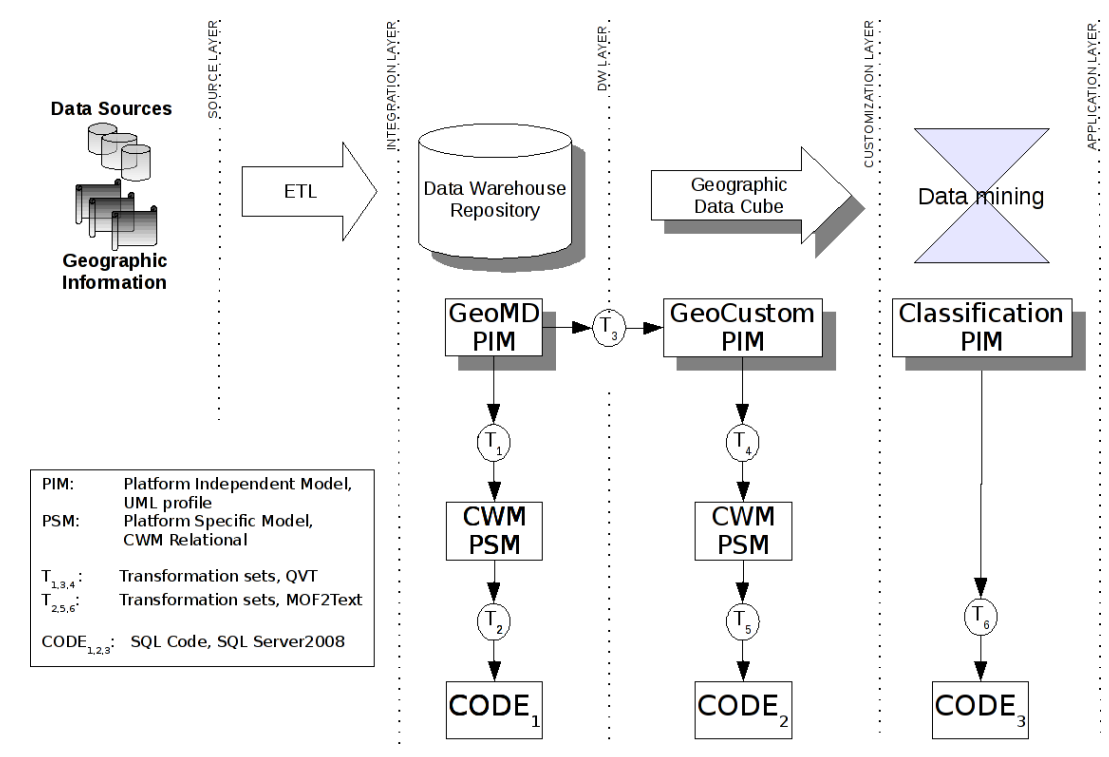

Fig. 1. Model driven framework for geographic knowledge discovery development

conceptual element for represent the geographic information in the multidimensional modeling with its own semantic and OCL restrictions. Thus, we have introduced a new element inspired on GIS layers, meaning a implementation of geographic information that describe a phenomenon inside a reference system. We have also introduced in this new element, a name, a geometric attribute and the possibility to add some descriptive attributes.

Finally, we have implemented new element in a UML profile by creating a new stereotype named Layer and represented by $\square$ icon. It also has an attribute named geometry that describes the geometric type of the objects represented by the layer. All the allowed geometric primitives have been grouped in a enumeration element named GeometricTypes. These primitives are included on $\mathrm{ISO}^{3}$ and $\mathrm{OGC}^{4}$ spatial standards, in this way we ensure the final maping to platform code. The resultant data model is, named GeoMD and is able to implement correctly the geographic information in the multidimensional structure (see Fig. 2).

\section{Geographic Querying Model}

The customization layer encourage the construction and delivering of a data cube for the different analysis tools inside the application layer. We refer as geographic customization to the process in which the user take advantages of GIS functionalities over a data cube. For example, suppose a data cube corresponding to "monthly total sales of pharmaceutical products in all supermarkets" and the possible geographic customized data cube corresponding to "monthly total sales of pharmaceutical products in all supermarkets located less than $500 \mathrm{~m}$ of a hospital". Therefore, in an initial model we should have the different geographic layers available (i.e.,

\footnotetext{
${ }^{3}$ International Organization for Standardization, http://www.iso.org

${ }^{4}$ Open Geospatial Consortium, http://www.opengeospatial.org
}

hospital locations, supermarket locations), this information could be easily taken from the repository model. Once we have the geographic information we should be able to establish conditions and relations so we will need topological relations (i.e., intersect, cross, inside, etc.), a distance relation and boolean operators to join different relations. Finally, another geometric object is required, that is a possible user defined area. The user could define an specific area that could be related with the other geographic objects in order to focus the analysis in this interesting area or just be involved in different geographic predicates.

In order to introduce this customization model into our framework, we implement it in a UML profile (GeoCustom PIM in Fig. 1). To represents the geographic information that the user could relate, we have defined a new element, it has been implemented by the ThematicLayer stereotype and represented by $\frac{\pi}{ \pm}$. Similar to spatial levels, it has a geometric attribute that describe the spatiality of the phenomenon. The allowed geometric primitives have been grouped in a enumeration element named GeometricTypes as we did it in GeoMD model of previous section. These primitives are included on ISO and OGC spatial standards, in this way we ensure the final mapping to platform code. To be able to represent user defined areas we introduce an element implemented by the UserDefinedArea stereotype and represented by (回). It also has a geometric description of interesting area and the possibility to add some descriptive attributes.

Finally, in order to relate all these geographic information, we define three kind of relations. The first one corresponds to a distance relation and it is implemented by an stereotype named DistanceRelation, it have an attribute (named distance) to define a boolean expression (i.e., less than 500 meters or < $500 \mathrm{~m}$.). The other relation element grouped all the others available topological operations, it is implemented in a stereotype named TopologicalRelation and it have an attribute (operator) 


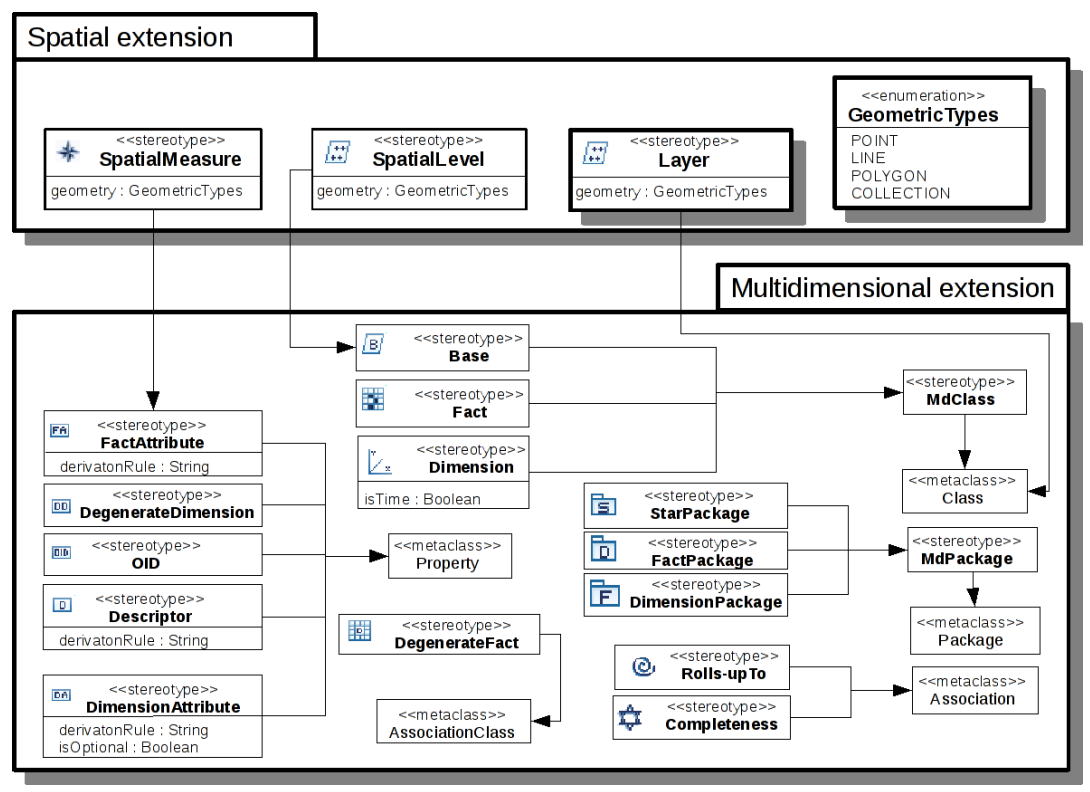

Fig. 2. Spatial multidimensional model implemented in a UML profile extension

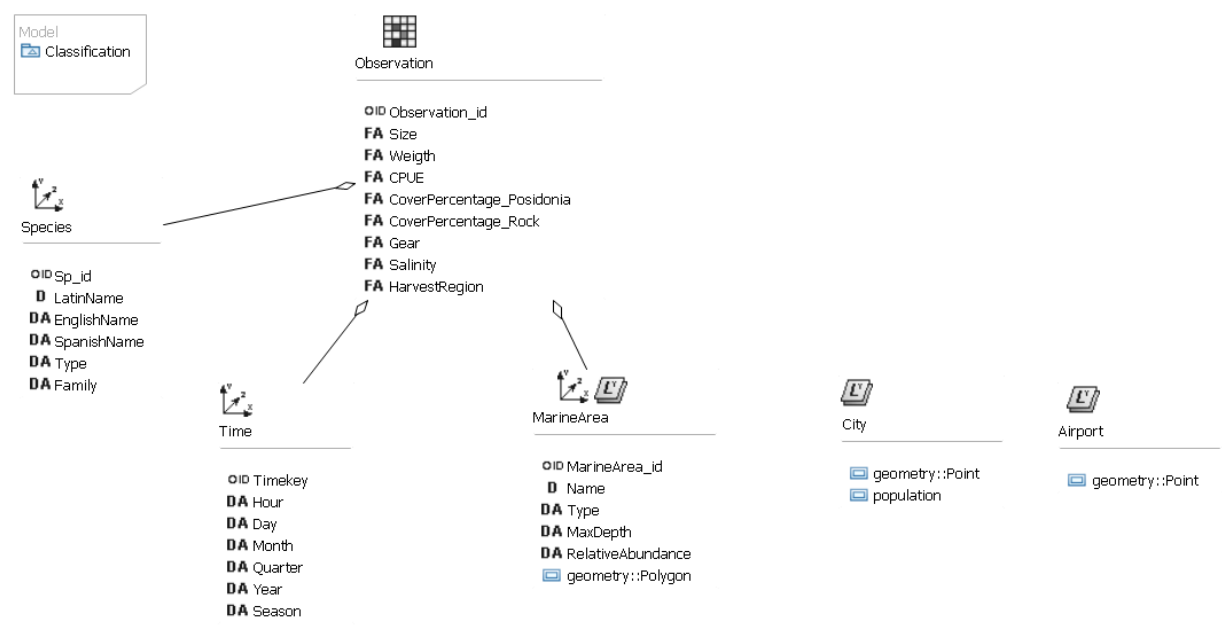

Fig. 3. Geographic query example using developed UML profile extension

that define the specific topological type of the relation. The most common operations are grouped in an enumeration named TopologicalTypes and more could be added easily. Finally, we have defined a boolean operation implemented in a association class stereotyped as BooleanRelation. It supports the most common boolean operators (and, or, not). In Fig 4 , it is shown a complete diagram of the UML profile extension presented.

\section{DATA Mining MOdel}

Data mining layer contains the knowledge discovery elements participating in the GKD. In this work, we show the use of the GKD with an example that analyzes several characteristics of Marine Areas to obtain a classification of "Relative Abundance". There are several non-geographical attributes such as Marine Area type (Protected or not), Catch

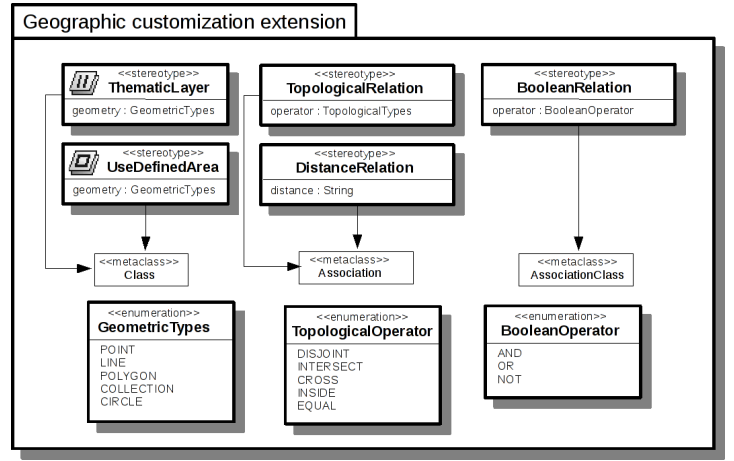

Fig. 4. Geographic customization model implemented in a UML profile extension 


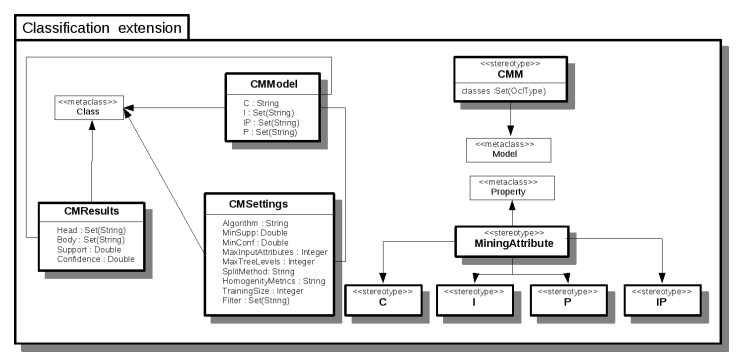

Fig. 5. Classification mining model implemented in a UML profile extension

Per Unit Effort (CPUE), Posidonia and Rock cover percentages, and other geographical data such as total marine area, maximum depth, perimeter of the marine area, distance to the next protected marine area or distance to the next city.

Such classification will serve to describe the current state of the studied Marine Areas and to serve as "diagnosis" tool for the behavior of other Marine Areas. The design process of the DW gives users a suitable knowledge of the domain and structures represented by the multidimensional model. The benefit is to use it to obtain "non-trivial, new and useful" information to support the decision-making. For the sake of simplicity, we will use a reduced version of the DW (Fig. 3). This contains one fact (Observation), and three dimensions (Species, Time and MarineArea). It also contains three layers describing airports, cities and marine areas. In such way, we can, for example, introduce in our analysis the distances beetwen marine areas, cities and airports. This DW stores historical observation data for different marine areas, at different times, for different species, and coming from diverse sources. Therefore, in order to discover classes in this MD model we follow three steps: first to select the case we want to analyze, second to select Input and Predict attributes, third to adjust the parameters that control the final decision tree. In this example, we will classify the RelativeAbundance attribute, based on the values observed for the other previously mentioned attributes. Thus, we set RelativeAbundance as Predict, while the CPUE, CoverPercentage_Posidonia, CoverPercentage_Rock, MarineArea. Type, Size, Perimeter, Distance_to_next_Marine_Area, Distance_to_next_City and MaxDepth attributes are set as Input, meaning that they will be used to classify the Predict attribute. The result of this mining process will give us a diagnosis tool for the status of a Marine Area on the basis of the previously enumerated variables. The situation is captured by Fig.? The designed mining model is self-descriptive. Users can design models based on their domain knowledge. No constraints are imposed on the number of models users can design.

\section{IMPLEMENTATION}

We have chosen SQL Server 2008 as our platform mainly because it allows the implementation of spatial data following OGC standards and the use of many different datamining techniques. Therefore, we have implemented, in a semiautomatic way, a spatio-multidimensional repository, an example of a user-customized geographic view and a mining

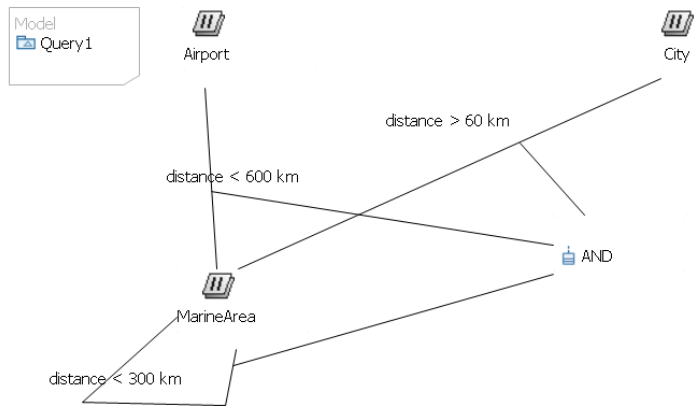

Fig. 6. Geographic query example using developed UML profile extension

$$
\begin{aligned}
& \text { Abundance Classification } \\
& \hline \text { 四arineArea_id } \\
& \square \text { MarineArea_Area } \\
& \square \text { CPUE } \\
& \square \text { CoverPercentage_Posidonia } \\
& \square \text { CoverPercentage_Rock }
\end{aligned}
$$

Minsupp $=10$ MaxInputattributes $=255$ MaxTreeLevels $=25$

HomogeneityMetrics=Entropy

Fig. 7. Classification model using developed UML profile extension

model for classification technique. Due to space constraints, we have briefly presented the scripts obtained following our methodology. Based on the MD model of the running example (Fig. MDschema), we have obtained a geographic query model and an example of a user-customized one (Fig. Customized). The code below shows the script deliver to SQL Server that creates the geographic view to be used next in the mining process.

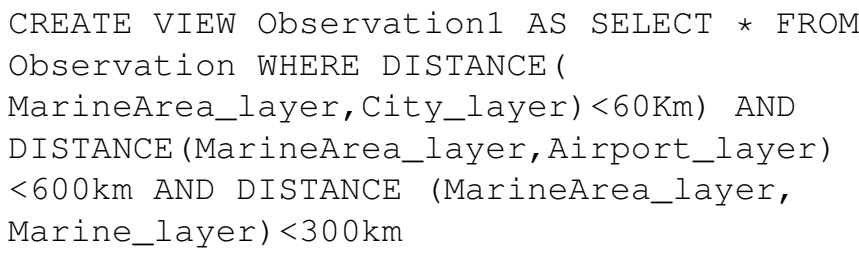

Based on the model used as Case study (Fig. DMCla), we have defined the Classification model, using RelativeAbundance as Predict_only, Observation_id as the nested table key, and CPUE, Size, CoverPercentage_Posidonia, CoverPercentage_Rock, MarineArea.Type, Area, Perimeter, Distance_to_next_Marine_Area, Distance_to_next_City and MaxDepth as Input. In SQL Server Predict means it will be used as Input and Predict, therefore we used Predict_only for RelativeAbundance. The code below shows the instruction that creates the mining model.

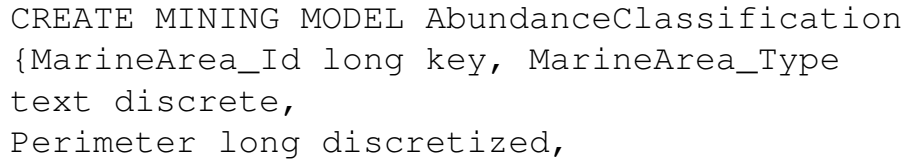




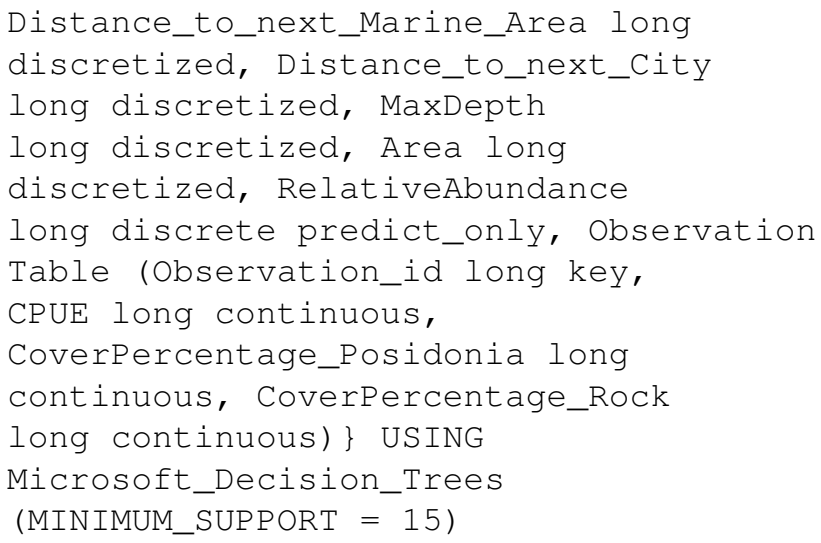

\section{CONCLUSION}

The main purpose of this work is to provide a modeling framework that address the development of the different part of a multilayer GKD process. The main advantages of our framework are (i) the dramatic reduction of the design effort due to automatics process are able to deliver specific platform code for the different process layers, (ii) the high quality systems obtained while good practices are reused in the different modeling tasks, (iii) the ability for adapting to new platform technologies while these tasks are entrusted to the modelto-model transformation scaffolding, and (iv) the suitability for the use of data mining techniques on geo-referenced data, avoiding isolated GIS and data mining session and supporting the documentation using intuitive visual models.

To accomplish this task, we have defined the spatial repository layer, the geographic customization layer and the application layer represented by different data mining techniques. Therefore, we have described how to build the different conceptual models by using the profile extension of the Unified Modeling Language (UML). In order to show the suitability of our propose, we have modeled an actual case of study that handles a very large database that contains geo-referenced data about the abundance of commercial fish species around marine protected areas. For this purpose, we have also developed an Eclipse-based prototype that implements our development framework. Finally, we have shown how the resulted models are implemented on a commercial spatial data base management server with data mining facilities such as Microsoft SQL Server 2008. As future work, we are developing UML metamodel extensions to represent other data mining techniques.

\section{ACKNOWLEDGEMENTS}

This work has been partially supported by the ESPIA project (TIN2007-67078) from the Spanish Ministry of Education and Science and by the QUASIMODO project (PAC08-01570668) from the Castilla-La Mancha Ministry of Education and Science (Spain). Octavio Glorio is funded by the University of Alicante under the $11^{\text {th }}$ Latin American grant program.

\section{REFERENCES}

[1] Sandro Bimonte, Anne Tchounikine and Maryvonne Miquel, "Geocube, a multidimensional model and navigation operators handling complex measures: Application in spatial olap," in Advances in Information Systems (ADVIS). Berlin / Heidelberg, Germany: Springer, 2006, pp. $100-109$.

[2] S. Bimonte, P. Wehrle, A. Tchounikine, and M. Miquel, "Gewolap: A web based spatial olap proposal," in OTM Workshops (2), 2006, pp. 1596-1605.

[3] S. Bimonte, A. Tchounikine, and M. Bertolotto, "Integration of geographic information into multidimensional models," in $\operatorname{ICCSA}(1), 2008$, pp. 316-329.

[4] L. Gomez, S. Haesevoets, B. Kuijpers, and A. A. Vaisman, "Spatial aggregation: Data model and implementation," CoRR, vol. abs/0707.4304, 2007.

[5] A. Escribano, L. Gomez, B. Kuijpers, and A. A. Vaisman, "Piet: a gisolap implementation," in DOLAP '07: Proceedings of the ACM tenth international workshop on Data warehousing and OLAP. New York, NY, USA: ACM, 2007, pp. 73-80.

[6] R. do Nascimento Fidalgo, V. C. Times, J. da Silva, and F. da Fonseca de Souza, "Geodwframe: A framework for guiding the design of geographical dimensional schemas," in DaWaK, 2004, pp. 26-37.

[7] V. Times, R. Fidalgo, R. Fonseca, J. Silva, and A. Oliveira, A Metamodel for the Specification of Geographical Data Warehouses. Springer, 2009.

[8] J. da Silva, V. C. Times, A. C. Salgado, C. Souza, R. do Nascimento Fidalgo, and A. G. de Oliveira, "A set of aggregation functions for spatial measures," in DOLAP, 2008, pp. 25-32.

[9] J. da Silva, A. S. C. Vera, A. G. de Oliveira, R. do Nascimento Fidalgo, A. C. Salgado, and V. C. Times, "Querying geographical data warehouses with geomdql," in SBBD, 2007, pp. 223-237.

[10] O. Glorio and J. Trujillo, "An MDA Approach for the Development of Spatial Data Warehouses," in DaWaK, Turin, Italy, 2008, pp. 23-32.

[11] S. Luján-Mora, J. Trujillo, and I.-Y. Song, "A uml profile for multidimensional modeling in data warehouses," Data Knowl. Eng., vol. 59, no. 3, pp. 725-769, 2006. 